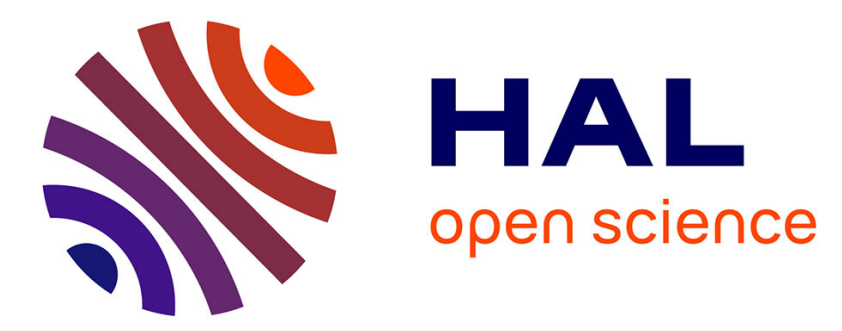

\title{
Low temperature epitaxial growth of $\mathrm{GaP}$ on Si by atomic-layer deposition with plasma activation
}

\author{
A Uvarov, A Gudovskikh, V Nevedomskiy, Artem Baranov, D Kudryashov, I \\ Morozov, Jean-Paul Kleider
}

\section{- To cite this version:}

A Uvarov, A Gudovskikh, V Nevedomskiy, Artem Baranov, D Kudryashov, et al.. Low temperature epitaxial growth of $\mathrm{GaP}$ on $\mathrm{Si}$ by atomic-layer deposition with plasma activation. Journal of Physics D: Applied Physics, 2020, 53 (34), pp.345105. 10.1088/1361-6463/ab8bfd . hal-02946512

\section{HAL Id: hal-02946512 https://hal.science/hal-02946512}

Submitted on 3 Nov 2020

HAL is a multi-disciplinary open access archive for the deposit and dissemination of scientific research documents, whether they are published or not. The documents may come from teaching and research institutions in France or abroad, or from public or private research centers.
L'archive ouverte pluridisciplinaire HAL, est destinée au dépôt et à la diffusion de documents scientifiques de niveau recherche, publiés ou non, émanant des établissements d'enseignement et de recherche français ou étrangers, des laboratoires publics ou privés. 
ACCEPTED MANUSCRIPT

\section{Low temperature epitaxial growth of GaP on Si by atomic-layer deposition with plasma activation}

To cite this article before publication: Alexander V. Uvarov et al 2020 J. Phys. D: Appl. Phys. in press https://doi.org/10.1088/1361-6463/ab8bfd

\section{Manuscript version: Accepted Manuscript}

Accepted Manuscript is "the version of the article accepted for publication including all changes made as a result of the peer review process, and which may also include the addition to the article by IOP Publishing of a header, an article ID, a cover sheet and/or an 'Accepted

Manuscript' watermark, but excluding any other editing, typesetting or other changes made by IOP Publishing and/or its licensors"

This Accepted Manuscript is @ 2020 IOP Publishing Ltd.

During the embargo period (the 12 month period from the publication of the Version of Record of this article), the Accepted Manuscript is fully protected by copyright and cannot be reused or reposted elsewhere.

As the Version of Record of this article is going to be / has been published on a subscription basis, this Accepted Manuscript is available for reuse under a CC BY-NC-ND 3.0 licence after the 12 month embargo period.

After the embargo period, everyone is permitted to use copy and redistribute this article for non-commercial purposes only, provided that they adhere to all the terms of the licence https://creativecommons.org/licences/by-nc-nd/3.0

Although reasonable endeavours have been taken to obtain all necessary permissions from third parties to include their copyrighted content within this article, their full citation and copyright line may not be present in this Accepted Manuscript version. Before using any content from this article, please refer to the Version of Record on IOPscience once published for full citation and copyright details, as permissions will likely be required. All third party content is fully copyright protected, unless specifically stated otherwise in the figure caption in the Version of Record.

View the article online for updates and enhancements. 


\title{
Low temperature epitaxial growth of GaP on Si by atomic-
}

\section{layer deposition with plasma activation}

\author{
A.V. Uvarov ${ }^{1}$, A.S. Gudovskikh ${ }^{1,2 *}$, V.N. Nevedomskiy ${ }^{3}$, A. I. Baranov ${ }^{1}$, D. A.
}

Kudryashov $^{1}$, I. A. Morozov ${ }^{1}$, J.-P. Kleider ${ }^{4}$

${ }^{1}$ St.Petersburg National Research Academic University RAS, St. Petersburg, 194091, Russia E-mail: gudovskikh@spbau.ru

${ }^{2}$ Saint-Petersburg Electrotechnical University “LETI”, 197376, Saint-Petersburg, Russia ${ }^{3}$ Ioffe Institute, 194021 St.-Petersburg, Russia

${ }^{4}$ GeePs, Group of electrical engineering - Paris, CNRS, CentraleSupélec, Univ. ParisSud, Université Paris-Saclay, Sorbonne Université, 91192 Gif-sur-Yvette Cedex, France Keywords: atomic layer deposition, gallium phosphide, plasma

\begin{abstract}
An approach for epitaxial growth of $\mathrm{GaP}$ layers on $\mathrm{Si}$ substrates at low temperature $\left(380^{\circ} \mathrm{C}\right)$ by plasma-enhanced atomic layer deposition (PEALD) is explored. A significant improvement of the crystalline properties of the GaP layers is obtained using additional in-situ Ar plasma treatment. The epitaxial growth for the first $20-30 \mathrm{~nm}$ of $\mathrm{GaP}$ on $\mathrm{Si}$ is demonstrated from transmission electron microscopy. Moreover, the use of in-situ Ar plasma treatment during the PEALD process allows one to increase the growth rate per cycle from $0.9 \pm 0.1 \AA$ /cycle to $1.9 \pm 0.1 \AA$ /cycle and reduce the RMS roughness from $3.76 \mathrm{~nm}$ to $1.88 \mathrm{~nm}$. The effect of Ar plasma treatment on the electronic properties of the GaP/Si interface is studied by deep level transient spectroscopy (DLTS). A defect level at $(0.33 \pm 0.03) \mathrm{eV}$ below the conduction band is observed in the subsurface layer of $\mathrm{Si}$ for the $\mathrm{GaP} / \mathrm{Si}$ structure grown under Ar plasma treatment. However, the defect response observed by DLTS vanishes after rapid thermal annealing at $500^{\circ} \mathrm{C}$ in nitrogen ambient.

Submitted to Journal of Physics D: Applied Physics
\end{abstract}




\section{Introduction}

Gallium phosphide (GaP) epitaxially grown on $\mathrm{Si}$ is of great interest for the integration of III-V and Si technologies. GaP being a III-V semiconductor with a band gap of $2.26 \mathrm{eV}$ and having a lattice mismatch with Si of less than $0.4 \%$ may be used as a nucleation layer for further growth of III-V compounds or as a window/emitter layer for silicon based solar cells [1-3]

Epitaxial growth of GaP layers on $\mathrm{Si}$ substrates may be achieved by metal-organic chemical vapor epitaxy (MOVPE) [4] or molecular beam epitaxy (MBE) [5]. Several important problems should be solved for the growth of polar III-V materials on non-polar Si substrates. The nucleation phase of III-V materials may give rise to bonds between two group-III or two group-V atoms. These bonds between similar atoms act as a boundary between two single crystalline domains opposite in phase (antiphase boundaries), which could propagate along the direction of growth. To improve the crystalline properties of the GaP layer the time modulated procedure was proposed recently, where deposition of a Ga monolayer is followed by that of a P one for the GaP nucleation stage. The terms atomic layer epitaxy (ALE) for MOVPE [6-8] and mobility enhanced epitaxy (MEE) for MBE [8] were used to describe this procedure, which could significantly enhance the quality of the epitaxy. However, high temperatures, above $600{ }^{\circ} \mathrm{C}$, used for $\mathrm{Si}$ surface deoxidation and reconstruction as well as for the growth process lead to a significant decrease in the lifetime of minority charge carriers in silicon substrates, which negatively affects the resulting efficiency of solar cells [9, 10].

We propose to use a plasma deposition technique to provide epitaxial growth of III-V compounds on $\mathrm{Si}$ at low temperature. This is possible due to the fact that plasma energy is locally scattered by surface atoms and can enhance the rate of surface reactions and the surface diffusion of adatoms [11]. To gain the advantage of the time modulated process (ALE or MEE) developed for nucleation of the epitaxial layers the atomic layer deposition (ALD) approach is suggested to be used in combination with plasma stimulation. Plasma-enhanced atomic layer deposition (PEALD) has several advantages such as high-quality uniform deposition on large 
areas, conformal layer growth on textured surfaces, trenches and holes with a high aspect ratio. Moreover, PEALD can be used to grow thin layers of material over large areas with high throughput, which is one of the main advantages for photovoltaic applications.

The possibility to obtain microcrystalline $\mathrm{GaP}$ by a PEALD-like method with a continuous hydrogen plasma was previously shown [12]. However, hydrogen plasma of high power used in those processes to increase the crystallinity of GaP leads to damages in the $\mathrm{Si}$ subsurface layer, which results in a drop of the photovoltaic performance [13]. Thus, a way to provide epitaxial growth of $\mathrm{GaP}$ at low temperature without deteriorating the electronic properties of the silicon substrate is an important issue. Recently, the use of in-situ annealing in argon plasma was shown to improve the crystalline quality to the epitaxial level of aluminum nitride layers obtained by PEALD [14]. Here we explore the possibility to obtain thin epitaxial GaP layers using PEALD at temperatures below $400^{\circ} \mathrm{C}$ using in-situ annealing in Ar plasma.

\section{Experiment details}

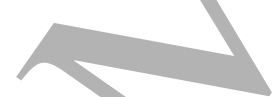

The process of ALD growth of GaP was realized using the Oxford Plasmalab 100 PECVD setup with a capacitively coupled plasma reactor. Trimethylgallium (TMG) and phosphine $\left(\mathrm{PH}_{3}\right)$ were used as the precursors of gallium and phosphorus, respectively. Hydrogen $\left(\mathrm{H}_{2}\right)$ was used as a carrier gas for TMG providing a precursor flow into the chamber using a bubbling system. The growth surface was alternatively submitted to TMG and $\mathrm{PH}_{3}$ flows. To provide $\mathrm{PH}_{3}$ decomposition at low temperature an $\mathrm{RF}$ (13.56 MHz) plasma was used at the phosphorous deposition/step. After exposure to each precursor, the chamber was purged with argon to avoid mixing precursors and parasitic CVD growth. Additionally, a modified process was also explored, where an Ar plasma treatment step was introduced immediately before the TMG step. The main deposition parameters are presented in Table 1. 
Table 1. Process parameters of GaP deposition by PEALD

\begin{tabular}{llllll}
\hline ParameterlStep & Gallium & Purge & Phosphorus & Purge & Ar plasma \\
\hline Gas composition & $5 \% \mathrm{TMG} / \mathrm{H}_{2}$ & $\mathrm{Ar}$ & $\mathrm{PH}_{3} / \mathrm{H}_{2}$ & $\mathrm{Ar}$ & $\mathrm{Ar}$ \\
Time, $\mathrm{s}$ & 5 & 10 & 3 & 10 & $0-15$ \\
Dose, nanomoles $/ \mathrm{cm}^{2}$ & $5-50$ & - & $0.5-3.7$ & - & - \\
Pressure, mTorr & 350 & 0 & 350 & 0 & 350 \\
RF power density, $\mathrm{mW} / \mathrm{cm}^{2}$ & 0 & 0 & 90 & 0 & 90 \\
\hline
\end{tabular}

GaP layers were grown on $\mathrm{Si}(100)$ substrates with a $4^{\circ}$ misorientation toward [110] as conventionally used for epitaxy [15]. Si substrates were cleaned by the Shiraki method followed by an HF-dip removal of oxide prior to the GaP growth [16]. The layers were grown at the temperature of $380{ }^{\circ} \mathrm{C}$. The choice of the process temperature is determined by the requirement for the evaporation of excess phosphorus from the growth surface, which occurs at temperatures above $300{ }^{\circ} \mathrm{C}$. In this case the growth of only one monolayer of phosphorous atoms, which are bonded to $\mathrm{Ga}$, is expected during one cycle. On the other hand, TMG starts to thermally decompose at about $280^{\circ} \mathrm{C}$ and keeps at least one methyl group up to $480^{\circ} \mathrm{C}$ [17]. The deposition rate was estimated by determining the resulting layer thickness using laser ellipsometry at a wavelength of $632.8 \mathrm{~nm}$ (Horiba PZ2000) and step profilometry (Ambios XP) on selective chemical etching steps of GaP on the silicon surface. The structural properties of the samples were investigated by transmission electron microscopy (TEM) and electron diffraction (JEOL JEM 2100F) with $200 \mathrm{kV}$ acceleration voltage. Capacitance deep-level transient spectroscopy (DLTS) was applied to study the influence of plasma treatment and in particular the possible creation of defects in the subsurface region of the silicon wafer to explore possible defects in the silicon near to the GaP/n-Si interface. For this characterization technique, special structures with Schottky barriers were fabricated by vacuum evaporation of gold on the top of GaP layers through a mask with circular holes (diameter of $1 \mathrm{~mm}$ ). DLTS measurements were carried out 
using a Boonton 7200 capacitance meter and a Janis VPF 100 liquid nitrogen cryostat in the temperature range of $80-360 \mathrm{~K}$ at the following conditions: $V_{\text {init }}=-2 \mathrm{~V}, V_{\text {pulse }}=+2 \mathrm{~V}, t_{\text {pulse }}=50$ ms. The time rate window was varied in the range of $10-1000 \mathrm{~s}^{-1}$.

\section{Results and discussions}

For the ALD process without Ar plasma activation two series of 200 cycles were performed. In the first series the TMG dose was varied from 5 to $50 \mathrm{nmol} / \mathrm{cm}^{2}$ at a constant $\mathrm{PH}_{3}$ dose of $1.25 \mathrm{nmol} / \mathrm{cm}^{2}$. During the second one the $\mathrm{PH}_{3}$ dose was varied from 0.5 to 3.7 $\mathrm{nmol} / \mathrm{cm}^{2}$ at a constant TMG dose of $50 \mathrm{nmol} / \mathrm{cm}^{2}$. For both series the growth rate of GaP does not depend on the dose of TMG and $\mathrm{PH}_{3}$ and is equal to $0.9 \pm 0.1 \AA /$ cycle (Figure 1) being more than 2.5 times lower compared to one monolayer per cycle. The saturation of the growth rate, which is observed in the whole range of TMG and $\mathrm{PH}_{3}$ dose, means that a self-limitation occurs for both precursors, indicating that the ALD growth mode was achieved.

Next the effect of Ar plasma activation before the TMG step in each cycle on the deposition rate per cycle was investigated. The treatment time in Ar plasma was varied in the range of 0 $15 \mathrm{~s}$ at constant doses of TMG and $\mathrm{PH}_{3}$ of $12.5 \mathrm{nmol} / \mathrm{cm}^{2}$ and $1.25 \mathrm{nmol} / \mathrm{cm}^{2}$, respectively. The dependence of the growth rate on the duration of the argon plasma step is presented in Figure 1. First an increase of growth rate per cycle (GPC) with the argon plasma step duration $\left(t_{A r}\right)$ is observed. Then for $\mathrm{t}_{\mathrm{Ar}} \geq 10 \mathrm{~s}$ the GPC reaches saturation at the value of $1.9 \pm 0.1 \AA$ /cycle, which does not exceed one monolayer per cycle $(2.73 \AA$ \&/cycle $)$, being in compliance with the ALD regime. However, introduction of an additional Ar plasma step (200 W, 15 s) before the $\mathrm{PH}_{3}$ step does not lead to an increase of the growth rate. This is probably due to the fact that $\mathrm{PH}_{3}$ decomposition occurs at high RF plasma power $(200 \mathrm{~W})$, which provides significant energy to phosphorous atoms during this step. An influence of Ar plasma treatment at the previous step seems to be less important for phosphorous deposition. 


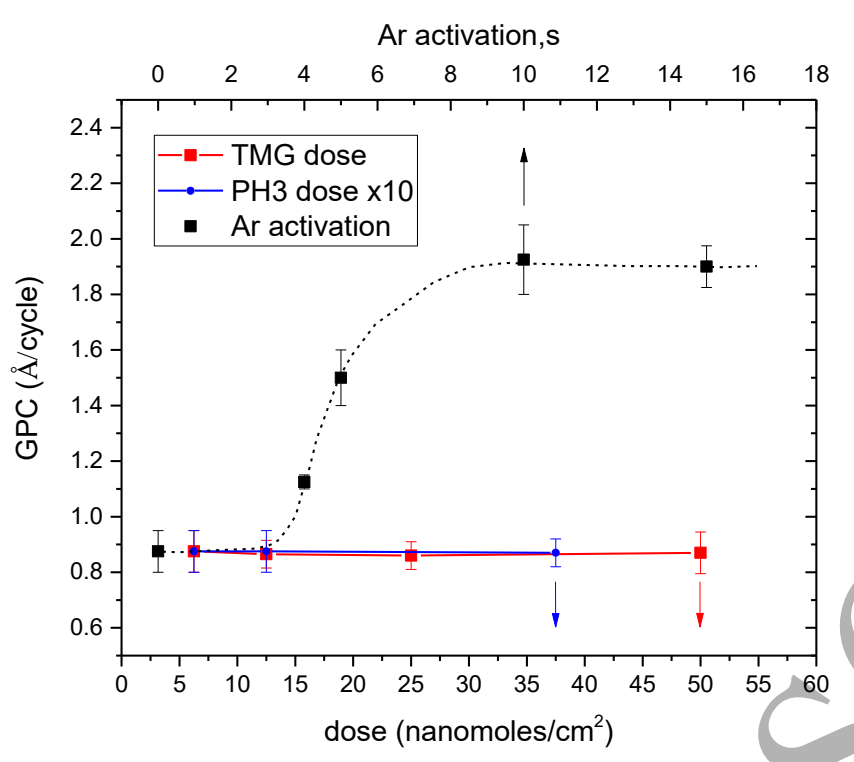

Figure 1. Growth rate per cycle (GPC) as a function of TMG and $\mathrm{PH}_{3}$ doses and dependence on Ar plasma step duration.

Along with the increase of the deposition rate due to the Ar plasma treatment, a significant decrease of the surface roughness of the layers was observed by atomic force microscopy (AFM) (Figure 2). AFM measurements were first performed for samples with the same thickness. Thus, 400 cycles of PEALD without Ar plasma and 200 cycles with Ar plasma step duration of $15 \mathrm{~s}$ were used to obtain two GaP layers of about $40 \mathrm{~nm}$. The RMS roughness decreases from $3.76 \pm 0.1 \mathrm{~nm}$ for the layer grown without Ar plasma to $1.88 \pm 0.1 \mathrm{~nm}$ for the sample prepared with Ar plasma. However, the roughness of the deposited films depends on the thickness. The GaP layer deposited without Ar plasma during 200 cycles (thickness of about $20 \mathrm{~nm}$ ) exhibits RMS roughness of $1.55 \pm 0.1 \mathrm{~nm}$. For very thin layers, both modes produce similar smooth surface. Indeed, for samples processed using 20 cycles with Ar plasma treatment and samples processed using 40 cycles without Ar plasma, which both have a thickness of about $4 \mathrm{~nm}$, the RMS roughness was equally found in the range of $0.12 \pm 0.1 \mathrm{~nm}$ indicating smooth surface for initial growth.

Obviously, the Ar plasma treatment affects the structure of the GaP layers. Bright field TEM images presented in Figure 3 confirm the improved RMS roughness when the Ar plasma 
step is introduced in the PEALD process. Moreover, a strong difference in the structure of the $\mathrm{GaP}$ films can also be observed. Indeed, the layer obtained with the additional Ar plasma step is much denser and uniform compared to the one fabricated without Ar plasma treatment.
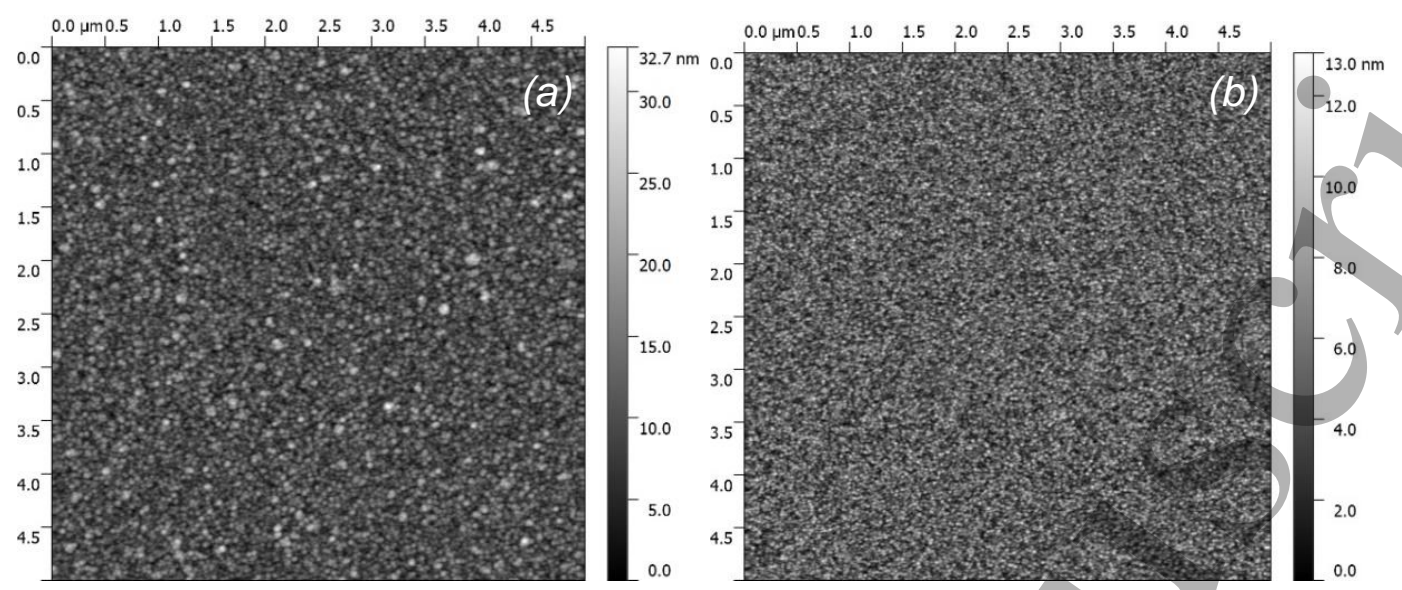

Figure 2. AFM images of GaP layers grown on Si by PE-ALD without and with $15 \mathrm{~s}$ Ar plasma treatment step.

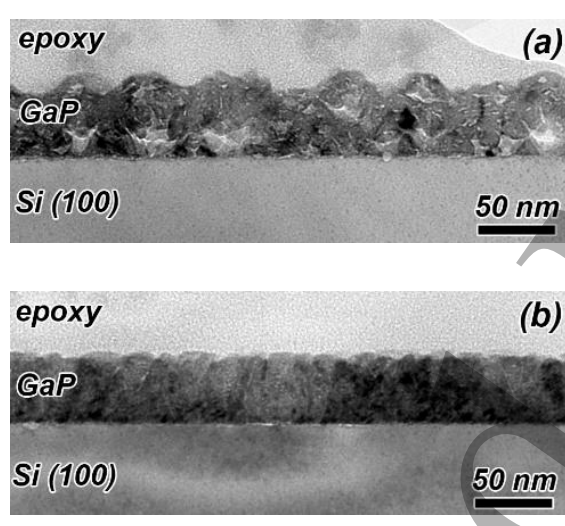

Figure 3. Cross section bright field TEM image of GaP on Si grown by PEALD without Ar plasma step (a) and with $15 \mathrm{~s}$ Ar plasma step (b).

Detailed studies of structural properties were performed using selective area electron diffraction (SAED) and high-resolution transmission electron microscopy (HRTEM). The SAED pattern is a superposition of the diffraction from a small part of single-crystal Si substrate, GaP layer and a small part of amorphous epoxy glue. The diffraction from the monocrystal Si substrate is a spot grid, while if the GaP layer is microcrystalline, the diffraction looks as a set of solid rings. The more the rings look like spots, the closer the GaP structure is to a single crystal. 
For the GaP layer obtained without Ar plasma the SAED pattern consists of almost solid circles, which indicates that the layer is microcrystalline with crystallites of different orientations (Figure 4a). The Dark field (DF) image obtained in part of $g=(111)$ ring indicates that the GaP crystallites of this orientation (bright contrast) are concentrated at the $\mathrm{GaP} / \mathrm{Si}$ interface (Figure 2b), which corresponds to initial epitaxial growth on the Si substrate. However, DF images taken from the other parts of the (111) plane (not presented) show separate $\mathrm{GaP}$ crystallites located in the middle-upper part of the GaP layer and having a different orientation. The presence of $10 \mathrm{~nm}$ size GaP crystallites with various orientations could also be observed in the HRTEM image presented in Figure 4c. A GaP epitaxial layer of a few nanometers could be observed by HRTEM at the GaP/Si interface, while further layer growth leads to deviations in the crystal orientation. Moreover, the thickness of the GaP epitaxial layer is nonuniform along $\mathrm{GaP} / \mathrm{Si}$ interface.

A detailed TEM study for the GaP/Si structures obtained by PEALD with the Ar plasma step demonstrates that the GaP layer is rather epitaxial (Figure 5). The diffraction pattern of epitaxial GaP layer, which exhibits marked spots, is clearly visible in the SAED pattern of the layer (Figure 5a). According to the dark-field image obtained in the part of $g=(111) \mathrm{GaP}-$ ring epitaxial growth of $\mathrm{GaP}$ is observed within the whole layer (Figure 5b). However, crystallites with different orientations could be observed 20-30 $\mathrm{nm}$ far from the GaP/Si interface, which is also confirmed by the rings in the diffraction pattern. The HRTEM image (Figure 5c) confirms epitaxial growth of GaP layer on the Si substrate. Substrate orientation is clearly reproduced by subsequent $\mathrm{GaP}$ growth (Figure 5d). Despite the high concentration of lattice defects like twins, misfits and threading dislocations, which could be observed by TEM in the epitaxial layer, a significant improvement of the crystalline properties was achieved using the in-situ Ar plasma treatment. 


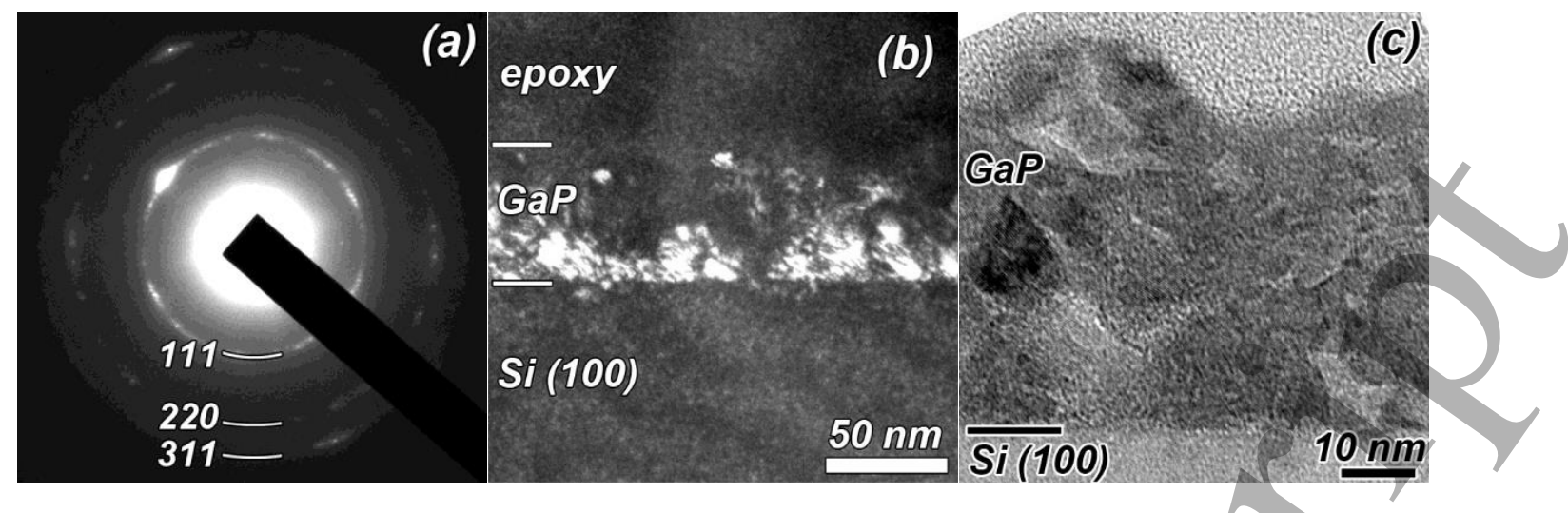

Figure 4. SAED pattern (a), DF image obtained in the part of $g=(111)$ ring $(b)$, and HRTEM image (c) of GaP layer grown by PE-ALD without Ar plasma.
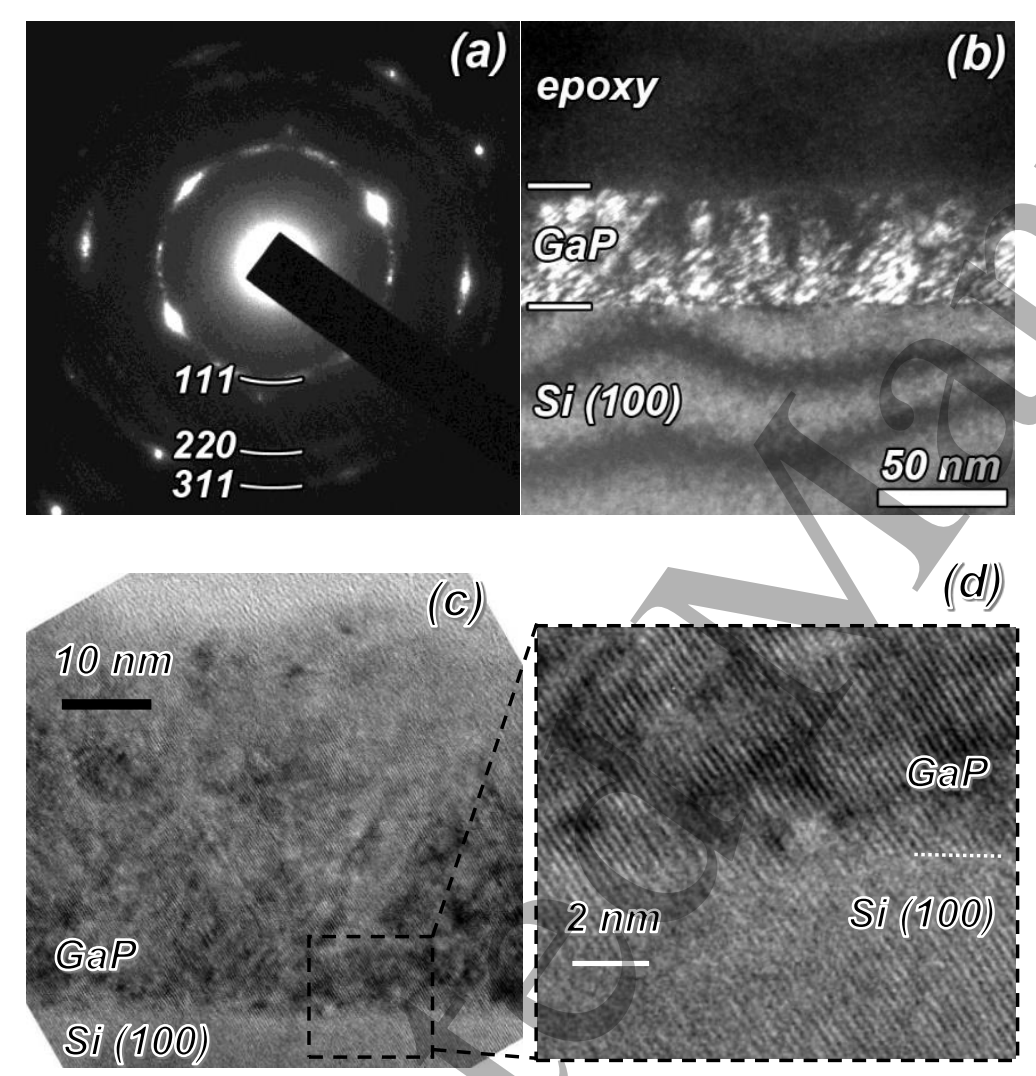

Figure 5. SAED pattern (a), DF image obtained in the part of $g=(111)$ ring (b), and HRTEM images $(c, d)$ of GaP layer grown by PE-ALD without Ar plasma.

The Ar plasma treatment introduced immediately before the TMG decomposition step influences both the growth rate and the structure of the layer. Both properties can be affected by the ion bombardment during Ar plasma treatment. Recently, the importance of substrate bombardment ion energy and flux on the film properties was demonstrated for the PEALD 
process [18]. The influence of the energy and dose of ions controlled by the substrate bias on the growth rate as well as on the crystalline properties was shown. In another work performed in the same Plasmalab setup as used here, it was shown that both Ar ion energy and flux increase with the RF power [19]. Energy of this ion flux bombardment during the Ar plasma treatment provides local heating of the growing surface as was proposed in Ref.14. Indeed, an increase of the surface temperature could lead to more effective TMG decomposition. According to ref. 17 in the temperature range from $380^{\circ} \mathrm{C}$ to $480^{\circ} \mathrm{C}$ TMG starts to lose the second methyl group. Methyl groups of TMG provide steric hindrance of the growth rate, which is a main reason why in practice the GPC is normally lower than one monolayer per cycle [20]. The observed saturation of the GPC with the increase of Ar plasma treatment duration (Figure 1a) could indicate that a threshold temperature is reached when TMG loses the second methyl group. In addition, the local heating of the surface leads to enhanced surface migration of adatoms and, therefore, to the improvement of the structural properties as was observed for the growth of AlN by PEALD with an Ar plasma treatment [14].

However, any plasma treatment is known to be a potential source of radiation-induced defects, which in our case could be formed in the Si substrate. Previously, for the GaP growth performed with high power hydrogen plasma treatment, a damage of the Si substrate was observed by TEM and defects were detected at the GaP/Si interface by electrical measurements [13]. Here no damage of $\mathrm{Si}$ is observed by TEM (Figure $1 \mathrm{~b}$ ) at the GaP/Si interface, despite the short (3 s) treatment in $\mathrm{H}_{2}$ plasma during the $\mathrm{PH}_{3} / \mathrm{H}_{2}$ step and the long (15 s) Ar plasma treatment. However, to have a better assessment of the electronic properties of the GaP/Si interface, deep level transient spectroscopy (DLTS) measurements were performed on $\mathrm{GaP} / \mathrm{n}-\mathrm{Si}$ structures with a top Schottky barrier. DLTS spectra obtained for a rate window of $50 \mathrm{~s}^{-1}$ are presented in Figure 6. For the GaP/Si structure grown without Ar plasma treatment no responses are detected, while a clear defect response was observed in the range of 80-140 K for the sample fabricated with additional Ar plasma. Using the classical DLTS data treatment (see 
supplementary material) we could identify the defect level to be at $0.33 \mathrm{eV} \pm 0.03 \mathrm{eV}$ below the conduction band. Ar plasma is known to be a source of radiation-induced defects in Si during magnetron sputtering of ITO on a-Si:H/c-Si heterostructures due to hard UV radiation. However, thermal annealing at a temperature approximately one hundred degrees higher than the deposition temperature allows one to recover the carrier lifetime in the Si wafer [21]. Our $\mathrm{GaP} / \mathrm{Si}$ structure grown with the Ar plasma treatment step was annealed at $500{ }^{\circ} \mathrm{C}$ during 1 minute in nitrogen ambient. To verify that annealing of GaP layer does not affect the structural properties they were additionally monitored by Raman spectroscopy. No changes were observed for microcrystalline $\mathrm{GaP}$ films after annealing at the temperature of $500^{\circ} \mathrm{C}$ (see supplementary material). After annealing no defect response could be detected by DLTS (Figure 6). Thus, argon plasma leads to the formation of defects in the sub-surface region of the silicon wafer, however their concentration could be drastically reduced by rapid thermal annealing at $500^{\circ} \mathrm{C}$.

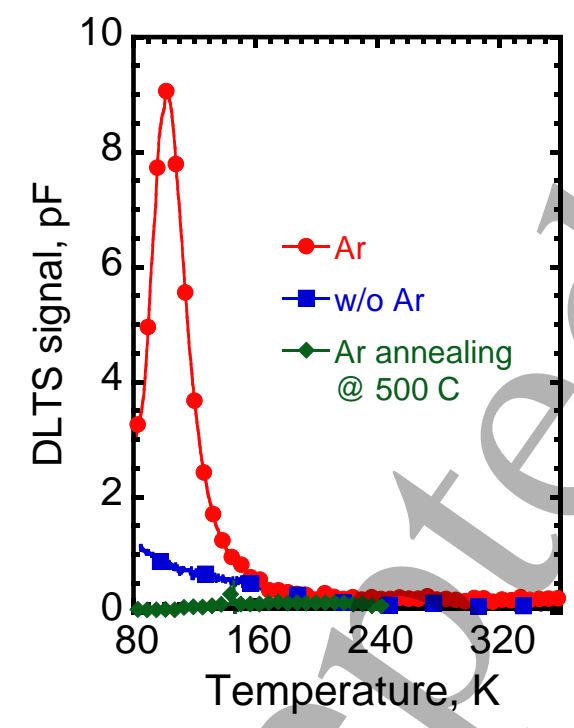

Figure 6. DLTS spectra (rate window of $50 \mathrm{~s}^{-1}$ ) for GaP layers grown with or without Ar plasma step, and also for a layer grown with the Ar plasma step and annealed at $500{ }^{\circ} \mathrm{C}$ during 1 minute in nitrogen ambient.

\section{Conclusion}


In conclusion, GaP epitaxial layers on silicon substrates were obtained by PEALD at a temperature of $380{ }^{\circ} \mathrm{C}$. It was shown that introducing an additional Ar plasma step during growth increases the growth rate per cycle and reduces the surface roughness due to an increase in the surface migration of adsorbed atoms. This effect has a saturation, which also indicates the compliance with the ALD regime and the absence of uncontrollable volumetric chemical reaction or re-evaporation of material from the chamber walls in the Ar plasma. The Ar plasma step also allows one to significantly improve the quality of epitaxial layers of $\mathrm{GaP}$ on $\mathrm{Si}$ substrates grown by PEALD. Finally, it leads to the formation of defects detected by DLTS in Si close to the GaP/Si interface, however these defects are suppressed by rapid thermal annealing at $500^{\circ} \mathrm{C}$.

\section{Acknowledgements}

This work was supported by the Russian Scientific Foundation under Grant № 17-19-01482. TEM characterization were performed using equipment owned by the Federal Joint Research Center "Material science and characterization in advanced technology" (id RFMEFI62119X0021).

\section{References}

[1] III-V Compound Semiconductors: Integration with Silicon-Based Microelectronics, (Eds:

T. Li, M. Mastro, A. Dadgar), CRC Press 2016.

[2] C. Zhang, N.N. Faleev, L. Ding, M. Boccard, M. Bertoni, Z. Holman, R.R. King, C.B. Honsberg, in 2016 IEEE 43rd Photovolt. Spec. Conf., 1950-1953, IEEE 2016.

[3] M. Feifel, J. Ohlmann, J. Benick, T. Rachow, S. Janz, M. Hermle, F. Dimroth, J. Belz, A. Beyer, K. Volz, D. Lackner, IEEE J. Photovoltaics, 2017, 7, 502.

[4] J.M. Olson, M.M. Al-Jassim, A. Kibbler, K.M. Jones, J. Cryst. Growth, 1986, 77, 515.

[5] Mahdad Sadeghi, Shumin Wang, J. Cryst. Growth, 2001, 227-228, 279.

[6] Y. Sakuma, K. Kodama, M. Ozeki, Appl. Phys. Lett. 1990, 56, 827. 
[7] J.R. Gong, S. Nakamura, M. Leonard, S.M. Bedair, N.A. El-Masry, J. Electron. Mater. 1992, 21, 965.

[8] T. Tsuji, H. Yonezu, M. Yokozeki, Y. Takagi, Y. Fujimoto, N. Ohshima, Jpn. J. Appl.Phys. A, 1997, 36, 5431.

[9] R. Varache, M. Darnon, M. Descazeaux, M. Martin, T. Baron, D. Muñoz, Energy Procedia, 2015, 77, 493.

[10] L. Ding, C. Zhang, T. U. Nærland, N. Faleev, C. Honsberg, M. I. Bertoni, Energy Procedia 2016, 92, 617 .

[11] A.J.M. Mackus, S.B.S. Heil, E. Langereis, H.C.M. Knoops, M.C.M. van de Sanden, W.M.M. Kessels, J. Vac. Sci. Technol. A Vacuum, 2010, 28, 77.

[12] A.S. Gudovskikh, A. V. Uvarov, I.A. Morozov, A.I. Baranov, D.A. Kudryashov, K.S. Zelentsov, A.S. Bukatin, K.P. Kotlyar, J. Vac. Sci. Technol. A, 2018, 36, 02 D408.

[13] A. S. Gudovskikh, A. V. Uvarov, I. A. Morozov, A. I. Baranov, D. A. Kudryashov, K.S. Zelentsov, A. Jaffre, S. Le Gall, A. Darga, A.Brezard-Oudot, J.P. Kleider, Phys. Status Solidi A, 2019, 216, 1800617.

[14] H.-Y. Shih, W.-H. Lee, W.-C. Kao, Y.-C. Chuang, R.-M. Lin, H.-C. Lin, M. Shiojiri, M.J. Chen, Sci. Rep. 2017, 7, 39717.

[15] T.J. Grassman, M.R. Brenner, S. Rajagopalan, R. Unocic, R. Dehoff, M. Mills, H. Fraser, S.A. Ringel, Appl. Phys. Lett. 2009, 94, 232106.

[16] A. Ishizaka, Y. Shiraki, J. Electrochem. Soc. 1986, 133, 666.

[17] F. Lee, T.R. Gow, R.I. Masel, J. Electrochem. Soc. 1989, 136, 2640.

[18] T. Faraz, H. C. M. Knoops, M. A. Verheijen, C. A. A. van Helvoirt, S. Karwal, A.Sharm Beladiya, A. Szeghalmi, D. M. Hausmann, Jon Henri, Mariadriana Creatore, and W. M. M. Kessels ACS Applied Materials \& Interfaces 2018, 10, 13158.

[19] D. Gahan, S. Daniels, C. Hayden, P. Scullin, D. O'Sullivan, Y.T. Pei, M.B. Hopkins, Plasma Sources Science and Technology 2012, 21, 024004. 
[20] Chemical Vapour Deposition. Precursors, Processes and Applications (Eds: A.C. Jones, M.L. Hitchman). Royal Society of Chemistry, Cambridge 2008.

[21] B. Demaurex, St. De Wolf, A. Descoeudres, Z. Ch. Holman, Ch.e Ballif, Appl. Phys. Lett. 2012, 101, 171604. 